\title{
Corrigendum to "Functional Stretching Exercise Submitted for Spastic Diplegic Children: A Randomized Control Study”
}

\author{
Mohamed Ali Elshafey, ${ }^{1}$ Adel Abd-Elaziem, ${ }^{2}$ and Rana Elmarzouki Gouda ${ }^{3}$ \\ ${ }^{1}$ Department of Physical Therapy for Growth and Developmental Disorder in Children and Its Surgery, \\ Faculty of Physical Therapy, Cairo University, Egypt \\ ${ }^{2}$ Faculty of Medicine, Zagazig University, Egypt \\ ${ }^{3}$ Physical Therapy Department, General Hospital of Mit Ghamr, Egypt \\ Correspondence should be addressed to Mohamed Ali Elshafey; elrahmapt@gmail.com
}

Received 14 April 2016; Accepted 31 May 2016

Copyright ( 2016 Mohamed Ali Elshafey et al. This is an open access article distributed under the Creative Commons Attribution License, which permits unrestricted use, distribution, and reproduction in any medium, provided the original work is properly cited.

In the article titled "Functional Stretching Exercise Submitted for Spastic Diplegic Children: A Randomized Control Study" [1], there was an error in Table 2, which should be corrected as follows: $7.6 \pm 7.32$ should be $76 \pm 7.32$. The revised table is below.

TABLE 2: Pretreatment comparison between right and left side in each group.

\begin{tabular}{|c|c|c|c|c|c|c|}
\hline \multirow[b]{2}{*}{ Variables } & \multicolumn{2}{|c|}{ Control group } & \multicolumn{4}{|c|}{ Study group } \\
\hline & $\begin{array}{c}\mathrm{RT} \\
\bar{X} \pm \mathrm{SD}\end{array}$ & $\bar{X}_{ \pm \mathrm{SD}}^{\mathrm{LT}}$ & $P$ value & $\begin{array}{c}\mathrm{RT} \\
\bar{X} \pm \mathrm{SD}\end{array}$ & $\begin{array}{c}\mathrm{LT} \\
\bar{X} \pm \mathrm{SD}\end{array}$ & $P$ value \\
\hline $\mathrm{H} \backslash \mathrm{M}$ ratio & $0.75 \pm 0.09$ & $0.77 \pm 0.07$ & $0.348^{*}$ & $0.76 \pm 0.07$ & $0.75 \pm 0.01$ & $0.792^{*}$ \\
\hline Popliteal angle & $77.2 \pm 5.58$ & $76.93 \pm 5.63$ & $0.77^{*}$ & $76 \pm 7.32$ & $76.3 \pm 7.56$ & $0.313^{*}$ \\
\hline Stride length & $79.46 \pm 7.8$ & $81.13 \pm 5.82$ & $0.16^{*}$ & $75.46 \pm 19.5$ & $80.53 \pm 6.0$ & $0.269^{*}$ \\
\hline Stride speed & $0.56 \pm 0.09$ & $0.59 \pm 0.6$ & $0.234^{*}$ & $0.6 \pm 0.11$ & $0.59 \pm 0.085$ & $0.539^{*}$ \\
\hline Stance phase \% & $72.86 \pm 3.7$ & $71.66 \pm 3.19$ & $0.212^{*}$ & $71.33 \pm 4.16$ & $70.6 \pm 4$ & $0.469^{*}$ \\
\hline
\end{tabular}

$\bar{X} \pm$ SD: mean \pm standard deviation; $P$ : level of significance; ${ }^{*}$ nonsignificant.

\section{References}

[1] M. A. Elshafey, A. Abd-Elaziem, and R. E. Gouda, "Functional stretching exercise submitted for spastic diplegic children: A Randomized Control Study," Rehabilitation Research and Practice, vol. 2014, Article ID 814279, 7 pages, 2014. 


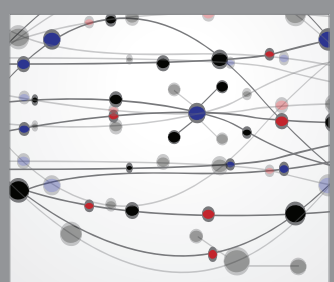

The Scientific World Journal
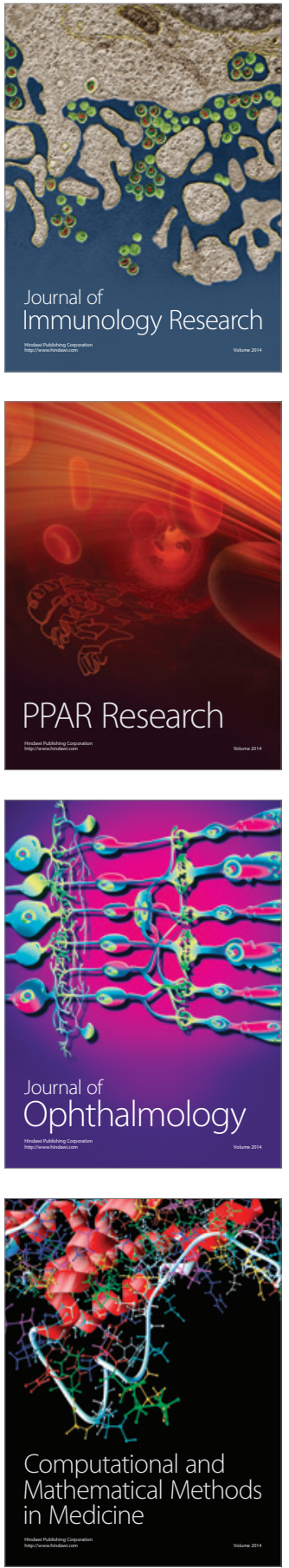

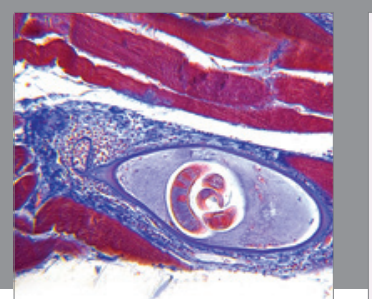

Gastroenterology Research and Practice

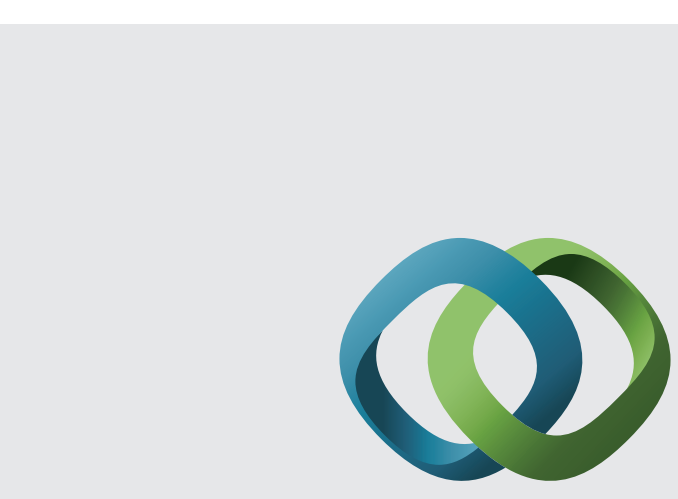

\section{Hindawi}

Submit your manuscripts at

http://www.hindawi.com
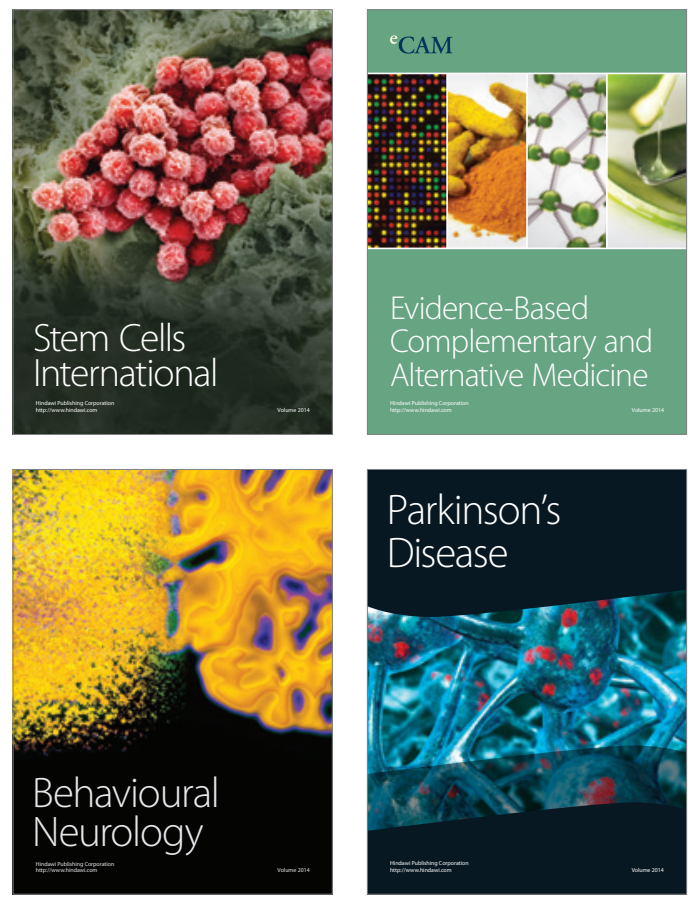
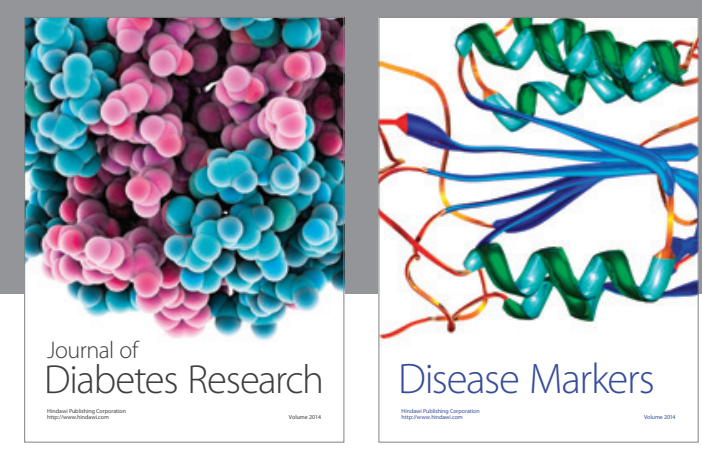

Disease Markers
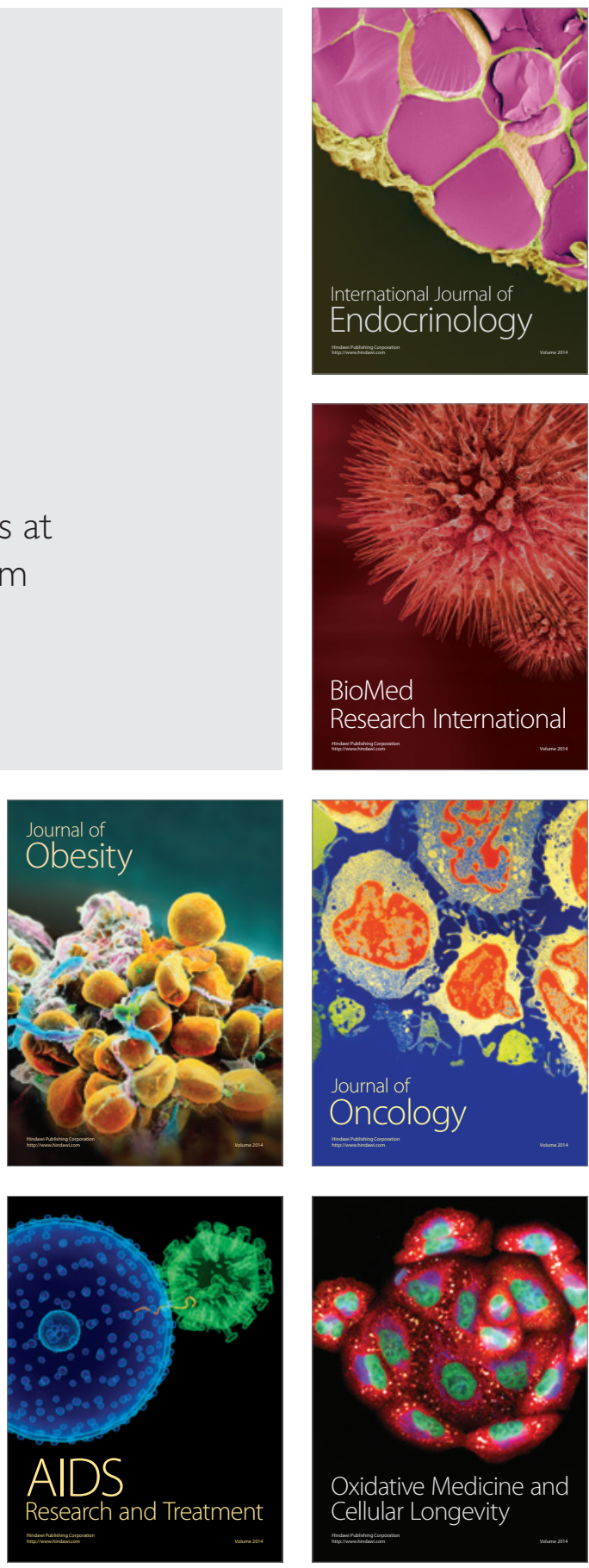